Supporting Information

\title{
Nano-Traffic Lights: Rayleigh Scattering Microspectroscopy of Optically Trapped Octahedral Gold Nanoparticles
}

Tatsuya SHOJI*1,2, Mamoru TAMURA ${ }^{3}$, Tatsuya KAMEYAMA ${ }^{4}$, Takuya IIDA ${ }^{3}$, Yasuyuki TSUBOI ${ }^{1,2}$, and Tsukasa TORIMOTO ${ }^{4}$

1) Division of Molecular Materials Science, Graduate School of Science, and

2) The OCU Advanced Research Institute for Natural Science and Technology (OCARINA), Osaka City University, 3-3-138 Sugimoto, Sumiyoshi, Osaka 558-8585, Japan

3) Department of Physical Science, Graduate School of Science, Osaka Prefecture University, 1-1 Gakuen-cho, Naka-ku, Sakai, Osaka 599-8531, Japan

4) Department of Materials Chemistry, Graduate School of Engineering, Nagoya University, Furo-cho, Chikusa-ku, Nagoya 464-8603, Japan 


\section{Analysis of Optically induced heating}

In steady state conditions, temperature elevation $\Delta T(\mathrm{r})$ at the surface of an optically trapped nanoparticle can be written as, ${ }^{1}$

$$
\Delta T(r)=\frac{Q}{4 \pi r \kappa}
$$

where $Q$ is the heat per unit time generated by light absorption of the nanoparticle [W], $r$ is the radial distance from the center of the particle, and $\kappa$ is the thermal conductivity of the surrounding medium $\left[\mathrm{W} \mathrm{K}^{-1} \mathrm{~m}^{-1}\right.$ ] (water $\kappa=0.60 \mathrm{~W} \mathrm{~K}^{-1} \mathrm{~m}^{-1}$ ). $Q$ is given as $Q=$ $I \sigma_{\mathrm{abs}}$, where $I=P / \pi w_{0}{ }^{2}$ is the laser light intensity $\left[\mathrm{W} / \mathrm{m}^{2}\right]\left(5.1 \times 10^{7} \mathrm{~W} / \mathrm{m}^{2}\right.$ corresponding to $50 \mathrm{~mW}$ of laser power $P$ when the beam radius $w_{0}$ was set to $500 \mathrm{~nm}$ ) and $\sigma_{\mathrm{abs}}$ is the absorption cross-section of the particle $\left[\mathrm{m}^{2}\right]$. The abso6rption cross-section $\sigma_{\mathrm{abs}}$ is defined as

$$
\sigma_{\mathrm{abs}}=\frac{2 \pi n_{m}}{\lambda} \operatorname{Im}\left[3 V \frac{\varepsilon_{p}-\varepsilon_{m}}{\varepsilon_{p}+2 \varepsilon_{m}}\right]
$$

where $n_{\mathrm{m}}$ is refractive index of the surrounding medium, $\varepsilon_{p}$ and $\varepsilon_{\mathrm{m}}$ is the dielectric constant of the particle and the medium $\left(\varepsilon_{\mathrm{p}}=-48+i 3.6, \varepsilon_{\mathrm{m}}=1.77\right.$ at $\left.1064 \mathrm{~nm}\right)$, respectively. Considering skin depth $\delta$ of the plasmonic nanoparticle ( $\delta=\lambda / 2 \pi \operatorname{Im}\left[n_{\mathrm{p}}\right], \lambda$ is the wavelength of the light, $n_{\mathrm{p}}$ is the refractive index of the particle) ( $\delta=24 \mathrm{~nm}$ at $\lambda=$ $1064 \mathrm{~nm})$, the particle volume $V$ is obtained from

$$
V=4 \pi \int_{0}^{a} r^{2} \exp [(r-a) / \delta] d r
$$

where $a$ is the radius of the nanoparticle [m] ( $a=50 \mathrm{~nm}$ when the particle shape is regarded as a sphere). Using this modeling, we calculated temperature $T(r)=T_{\infty}+\Delta T(r)$ $\left(T_{\infty}=298.15 \mathrm{~K}\right)$ as shown in Figure $\mathrm{S} 1 . \Delta T$ was estimated to be $10 \mathrm{~K}$ at $50 \mathrm{~mW}$. The photothermal heating (200 K/W) was good agreement with the previous literatures. ${ }^{1-4}$ 


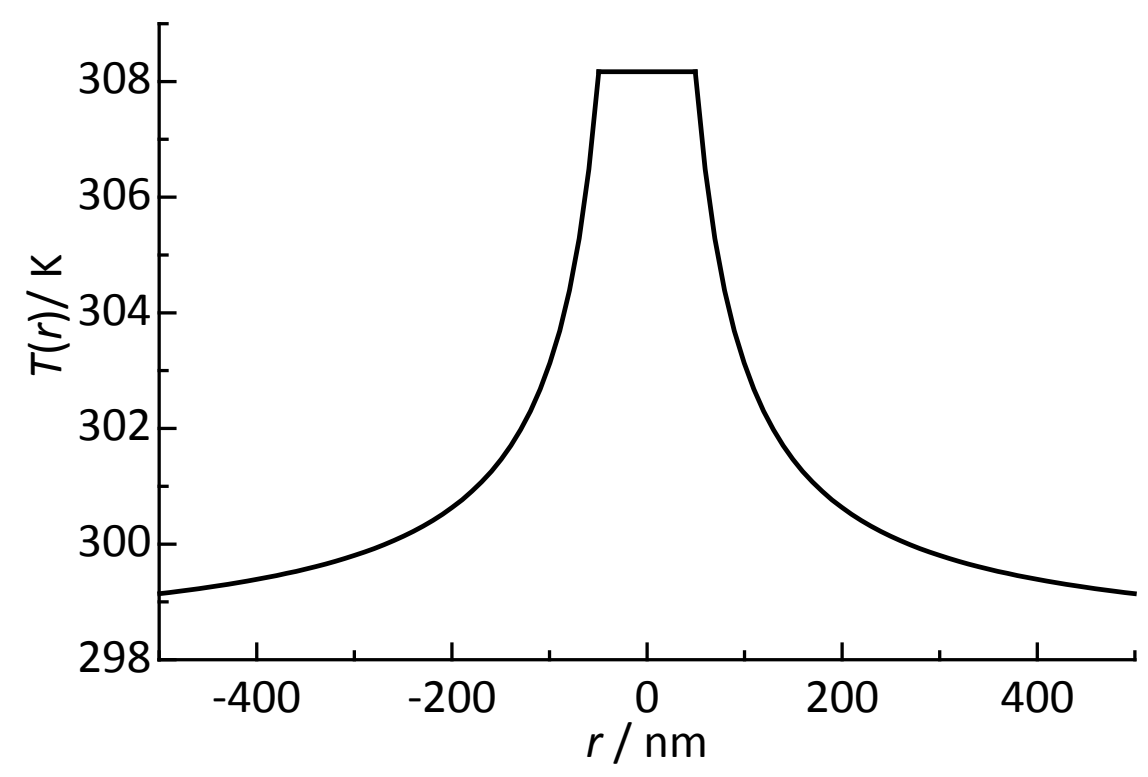

\section{Figure S1}

Spatial distribution of temperature $T(r)=T_{\infty}+\Delta T(r)\left(T_{\infty}=298.15 \mathrm{~K}\right)$ surrounding an optically trapped gold nanoparticles $(a=50 \mathrm{~nm})$ at $50 \mathrm{~mW}$.

\section{Numerical calculations for optical properties of a single octahedral gold nanoparticle}

In the manuscript, we discussed extinction spectra and spatial profiles of electric fields from a single octahedral gold nanoparticle (OGP) by changing the orientation of it in the electric field of light. The OGP allows some orientations including case (i) and (ii) (Fig. 5). For absorption (solid) and scattering cross sections (dashed line) (Fig. S2(a)), we carried out a finite difference time domain (FDTD) calculations of the OGP whose orientations (case (iii) and (iv)) were depicted in Fig. S2(b) together with the spatial distributions of enhanced electric field at 550 and 600 nm. The cross-sections from case (iii) were the almost same as those from case (i), while those from case (iv) were slightly red-shifted from case (i). 
(a)

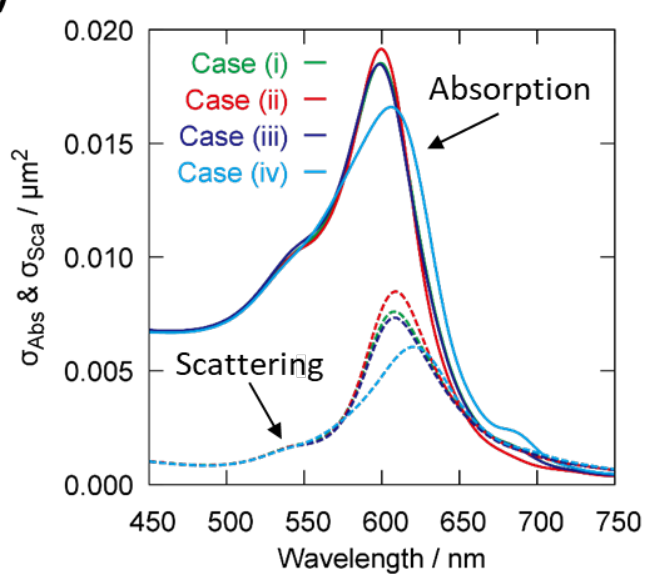

(b)

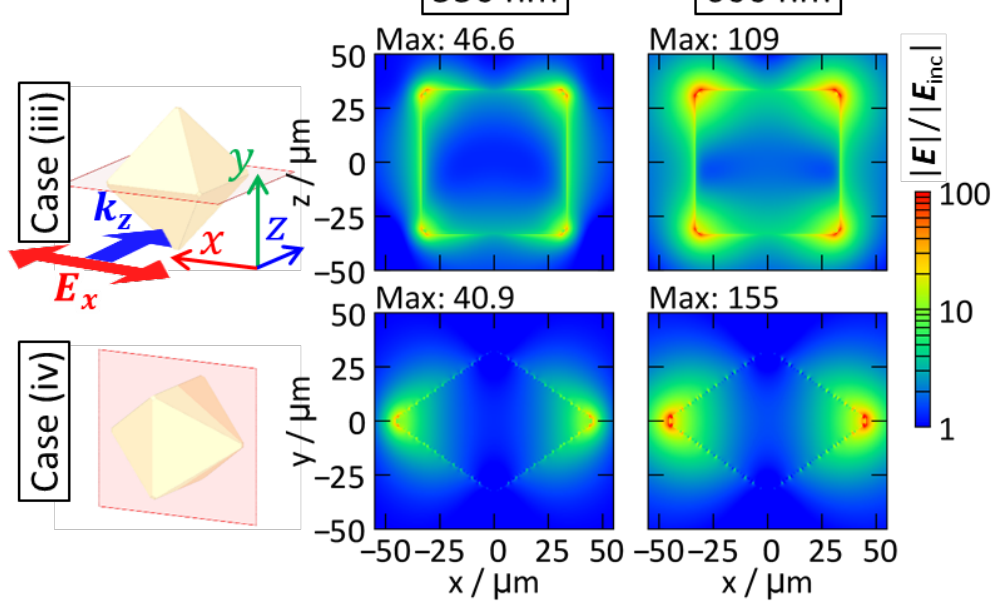

\section{Figure S2}

(a) Calculated absorption (solid lines) and scattering (dashed lines) cross-sections for an OGP dispersed in water. (b) The spatial distributions of enhancement factor of electric field $\left(|\boldsymbol{E}| /\left|\boldsymbol{E}_{\text {inc }}\right|, \boldsymbol{E}\right.$ is the enhanced electric field, and $\boldsymbol{E}_{\text {inc }}$ is the incident electric field) around an OGP at 550 and $600 \mathrm{~nm}$, where $\mathrm{x}-\mathrm{z}$ and $\mathrm{x}-\mathrm{y}$ distribution were depicted for case (iii) and case (iv), respectively, as indicated by the red frame in 3D model of (b). 


\section{Numerical calculations for optical properties of an OGP dimer with different gap distances}

For discussing the Rayleigh scattering spectra from an optically trapped OGP dimer (Fig. 4(d)), we carried out FDTD calculations for an OGP dimer with different gap distances; $30 \mathrm{~nm}, 16 \mathrm{~nm}, 10 \mathrm{~nm}, 4 \mathrm{~nm}$, and $2 \mathrm{~nm}$ (Fig. S3). We assumed a vertex-to-vertex configuration of an OGP dimer in water. Decreasing gap distance, a main scattering band was red-shifted to $626 \mathrm{~nm}, 639 \mathrm{~nm}, 653 \mathrm{~nm}, 692 \mathrm{~nm}$, and $736 \mathrm{~nm}$.

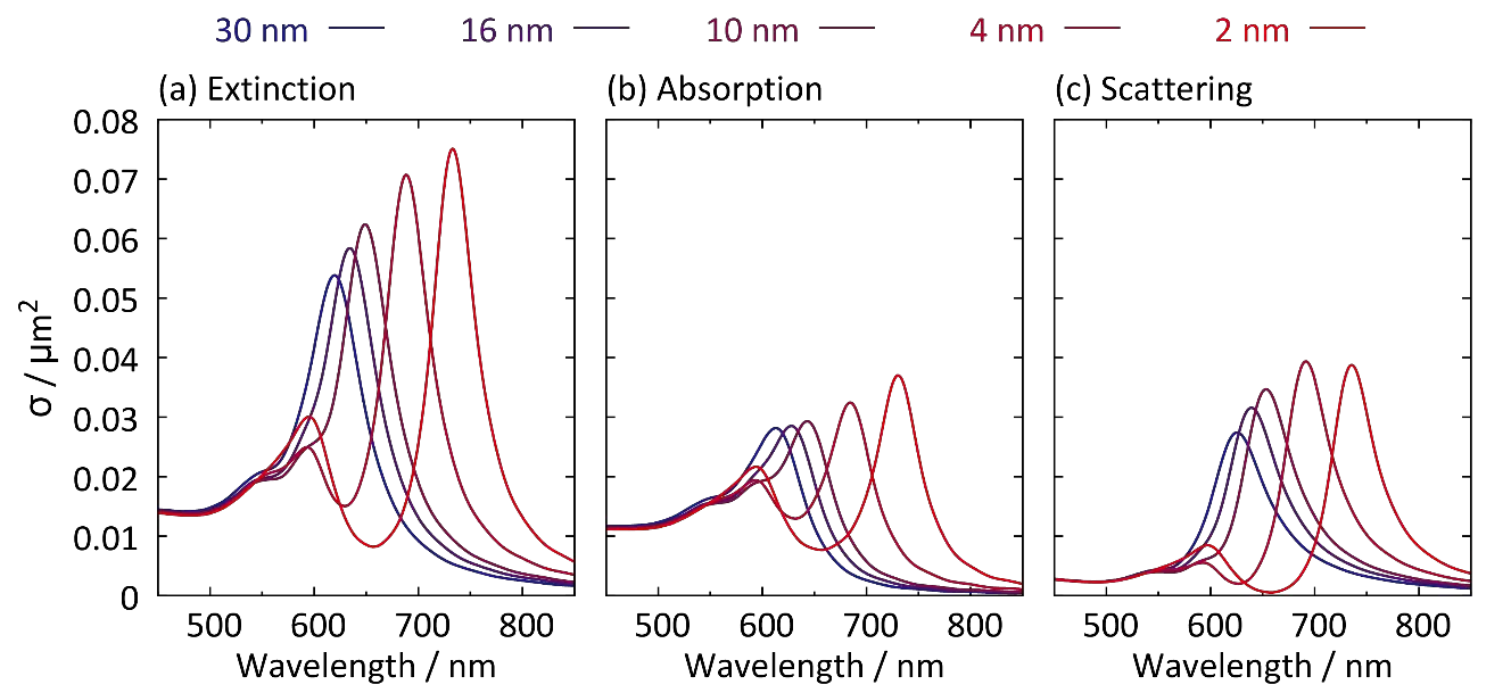

Figure S3

(a) Extinction, (b) absorption, and (c) scattering cross-sections of an OGP dimer with a gap distances: $30 \mathrm{~nm}, 16 \mathrm{~nm}, 10 \mathrm{~nm}, 4 \mathrm{~nm}$, and $2 \mathrm{~nm}$. The configuration of OGP dimer was vertex-to-vertex configuration described in the manuscript. 


\section{Supplementary movies}

Media 1: Brownian motion of OGPs in water under dark-field microscopy.

Media 2: Optical trapping of OGPs at $50 \mathrm{~mW}$ under dark-field microscopy.

\section{References}

(1) Melzer, J. E.; McLeod, E. Fundamental Limits of Optical Tweezer Nanoparticle Manipulation Speeds. ACS Nano 2018, 12, 2440-2447.

(2) Seol, Y.; Carpenter, A. E.; Perkins, T. T. Gold Nanoparticles: Enhanced Optical Trapping and Sensitivity Coupled with Significant Heating. Opt. Lett. 2006, 31, 2429-2431.

(3) Setoura, K.; Werner, D.; Hashimoto, S. Optical Scattering Spectral Thermometry and Refractometry of a Single Gold Nanoparticle under CW Laser Excitation. J. Phys. Chem. C 2012, 116, 15458-15466.

(4) Jauffred, L.; Taheri, S. M.-R.; Schmitt, R.; Linke, H.; Oddershede, L. B. Optical Trapping of Gold Nanoparticles in Air. Nano Lett. 2015, 15, 4713-4719. 